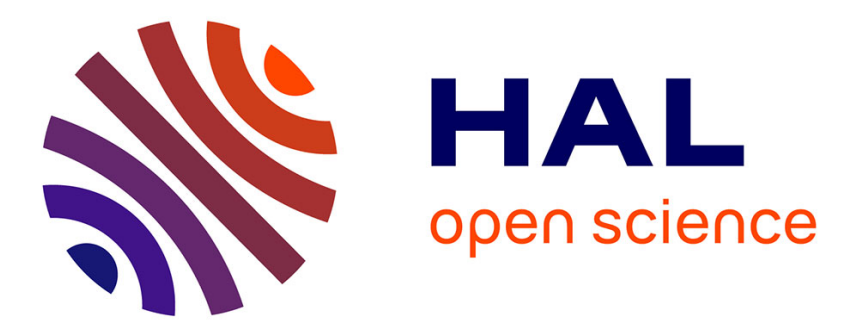

\title{
Sur la conservation de l'électricité et la conservation du poids dans les transformations des systèmes matériels
} M. Gouy

\section{To cite this version:}

M. Gouy. Sur la conservation de l'électricité et la conservation du poids dans les transformations des systèmes matériels. J. Phys. Theor. Appl., 1889, 8 (1), pp.227-230. 10.1051/jphystap:018890080022700 . jpa-00238946

\section{HAL Id: jpa-00238946 https://hal.science/jpa-00238946}

Submitted on 1 Jan 1889

HAL is a multi-disciplinary open access archive for the deposit and dissemination of scientific research documents, whether they are published or not. The documents may come from teaching and research institutions in France or abroad, or from public or private research centers.
L'archive ouverte pluridisciplinaire HAL, est destinée au dépôt et à la diffusion de documents scientifiques de niveau recherche, publiés ou non, émanant des établissements d'enseignement et de recherche français ou étrangers, des laboratoires publics ou privés. 


\title{
SUR LA CONSERTATION DE L'ÉLEGTRIGITÉ ET LA GONSERVATION DO POIDS DANS LES TRANSFORMATIONS DES SYSTÈMES MATÉRIELS;
}

\author{
PAR M. GOUY.
}

Je me propose de montrer comment ces deux lois générales (1) peuvent être rattachées au principe de Carnot.

Nous nous occuperons d'abord des actions électriques, en prenant comme point de départ expérimental la loi de Coulomb, qui exprime les actions mutuelles de conducteurs très petits, invariables, à température constante, isolés et électrisés, tels, par exemple, que les boules de la balance de Coulomb. De cette loi résulte la notion de masse électrique, considérée comme le coefficienl $m$ ou $m^{\prime}$ qui entre dans l'expression $\frac{m m^{\prime}}{r^{2}}$ de la force agissant entre deux de ces conducteurs. Dans ces conditions expresses, l'invariabilité de ce coefficient fait partie intégrante de la loi des actions électriques; nous pourrons donc considérer des masses électriques invariables, réalisées par de très petits conducteurs électrisés, qui sont et restent isolés et invariables, à température constante.

Considérons un système matéricl quelconque $\mathrm{S}$, électrisé et isolé. Nous admettrons qu'il existe de même, aux divers points de $\mathbf{S}$, des masses électriques $\mathbf{M}$ agissant suivant la loi précédente, mais qui peuvent varier, suivanı les transformations de $\mathrm{S}$, d'une manière inconnue. Nous allons montrer que la somme algébrique de ces masses, $\mathbf{\Sigma} \mathbf{M}$, demeure invariable.

Considérons une transformation quelconque de $\mathrm{S}$; désignons par A un quelconque des états par lesquels passe ce système. Soit $\theta$ le travail fourni, pendant la transformation, par des forces extérieures appliquées au système $\mathrm{S}$. Le travail $\theta$ peut être employé à produire en $\mathrm{S}$ tel effet thermique que l'on voudra, en sorte qu'il est inutile de considérer des sources de chaleur extérieures.

(1) Pour l'énoncé exact de la loi de conservation de l'électricité, et son ròle dans les théories électriques, je renverrai le lecteur au Mémoire de M. Lippmann (Journal de Physique, ${ }^{\text {e }}$ série, t. X, p. $38 \mathrm{r} ;$ r $88 \mathrm{r}$ ). 
Supposons que des masses électriques invariables $m$, définies comme plus haut, égales et en très grand nombre, mais telles que $\Sigma m$ ait une valeur déterminée, soient uniformément réparties sur une sphère de rayon $\mathrm{R}$ enfermant $\mathrm{S}$, et maintenues en place par des forces extérieures. Remarquons que les forces électriques produites par ces masses ont une résultante nulle en tous les points de $\mathrm{S}$; par suite, tout ce qui se passe en $\mathrm{S}$, et notamment le travail $\theta$, est indépendant de la présence des masses $m$.

Soit $R_{0}$ la valeur initiale de $R$ : nous ferons varier $R$ avant la transformation de $\mathrm{S}$, de $\mathrm{R}_{0}$ à $\mathrm{R}_{1}$, chacune des masses $m$ étant déplacée suivant un rayon de la sphère. $R$ restera ensuite invariable jusqu'à ce que $S$ ait atteint l'état $A$, el, pendant que $S$ est dans cet état, $R$ reviendra définitivement à sa valeur initiale $R_{0}$.

Soit $\theta^{\prime}$ le travail produit par les forces électriques agissant sur les masses $m$, à l'encontre des forces extérieures qui maintiennent ces masses. Le travail $d \theta^{\prime}$ produit quand $\mathrm{R}$ prend l'accroissement $d \mathrm{R}$ est

$$
d \theta^{\prime}=\frac{\Sigma m \Sigma \mathrm{M}}{\mathrm{R}^{2}} d \mathrm{R}(1) ;
$$

en désignant par M la masse électrique existant, à cet instant, en un point quelconque de $\mathrm{S}$, et en négligeant un terme dépendant des actions mutuelles des masses $m$, qui disparaittrait dans l'intégration. Il vient done

$$
0^{\prime}=\int_{\mathbf{R}_{0}}^{\mathrm{R}_{1}} \frac{\Sigma m \Sigma \mathrm{M}_{0}}{\mathbf{R}^{2}} d \mathbf{R}+\int_{\mathbf{R}_{1}}^{\mathbf{R}_{0}} \frac{\Sigma m \Sigma M_{1}}{\mathbf{R}^{2}} d \mathbf{R}=\Sigma m\left(\Sigma \mathbf{M}_{0}-\Sigma M_{1}\right)\left(\frac{\mathbf{I}}{\mathbf{R}_{0}}-\frac{\mathbf{I}}{\mathrm{R}_{1}}\right),
$$

en désignant par $M_{0}, M_{1}$ les masses électriques existant en un point quelconque de $\mathrm{S}$ dans l'état initial et dans l'état $\mathrm{A}$.

Supposons que l'on ait $\Sigma M_{0} \gtrless \Sigma M_{1}$. Comme nous pouvons disposer du signe de $\frac{1}{R_{0}}-\frac{1}{R_{1}}$, nous pouvons toujours faire que $\theta^{\prime}$ soit positif. $\Sigma m$ étant arbitraire, $\theta^{\prime}$ pourra être rendu aussi grand qu'on le voudra. Toute difficulté sur ce point disparaît si l'on remarque qu'on peut considérer, au lieu d'une seule sphère, $n$ sphères concentriques variant ensemble, en conservant leurs intervalles. Si

( ${ }^{1}$ ) On établit aisément cette formule par un calcul analogue à celui du flux de force. 
l'on suppose que l'écartement des masses $m$ est le même sur toutes ces sphères, le facteur $\frac{\Sigma m}{\mathrm{R}^{2}}$ dans l'expression de $d \theta^{\prime}$ aura la même valeur pour toutes, et par suite le travail total sera proportionnel à $n$, et pourra être accru sans limite.

Considérons maintenant l'ensemble du système $\mathrm{S}$ et des masses $m$. Cet ensemble constitue une machine à laquelle on fournit le travail $\theta$, et qui rend le travail $\theta^{\prime}$, sans l'intervention de sources de chaleur.

Si la transformation du système $S$ forme un cycle fermé, on ne peut pas avoir $\theta^{\prime}>\theta$, car il y aurait production de travail, ce qui est en opposition avec le principe de Carnot ( $\left.{ }^{1}\right)$. Or le travail $\theta^{\prime}$ peut être accru sans limite, à moins qu'on n'ait $\Sigma \mathbf{M}_{0}=\Sigma \mathbf{M}_{1}$. Il faut donc que cette égalité ait lieu, et, par suite, que l'on ait toujours $\Sigma M=$ const., puisque l'état A a été choisi arbitrairement ( $\left.{ }^{2}\right)$. C'est la loi de la conservation de l'électricité.

S'il est impossible de ramener le système $\mathrm{S}$ à son état initial, la production de travail par la machine idéale que nous considérons est rendue possible, au point de vue du principe de Carnot, par la modification de S. Mais, si l'on se refuse à admettre qu'une modification finie puisse suffire pour rendre possible la production d'une quantité illimitée de travail, la conclusion précédente subsiste.

( ${ }^{1}$ ) Mais non avec le principe de l'équivalence, si l'on regarde comme possible qu'un effet thermique se produise sur les masses $m$; le travail serait alors produit aux dépens de la chaleur du milieu ambiant. On peut démontrer que cet effet thermique n'a pas lieu si les forces électrostatiques sont indépendantes de la température; c'est sous cette forme que la démonsiration a été publiée précédemment [Sur la conservation de l'électricité et la Thermodynamique (Comptes rendus, 1888)]. La démonstration donnée ici est plus directe et indépendante de cette hypothèse; elle peut servir à en démontrer l'exactitude, en considérant comme transformation du système $\mathrm{S}$ l'échauffement d'un conducteur ćlectrisé qui constituerait ce système.

( ${ }^{2}$ ) Pour éviter toute difficulté, il convient de limiter la démonstration aux états A qui sont stables, et peuvent être maintenus aussi longtemps qu'on le veut. Dès lors, le déplacement des masses $m$ peut être opéré avec une vitcsse infiniment petite, et il n'y a pas lieu de s'occuper d'autre chose que des forces statiques, régies par la loi de Coulomb. De plus, au cas où un effet thermique se produirait sur les masses $m$ par lcur transport dans le champ, électrique ou magnétique, la température des petits conductcurs qui les supportent resterait alors égale à la température du milieu ambiant, supposée constante, et nous n'avons pas besoin de savoir si les forces électriques sont indépendantes de la tempirature. 
KORDA.

La même démonstration s'applique en général aux centres de forces agissant suivant la loi du carré des distances, et notamment à l'attraction newtonienne; $m$ et $\mathbf{M}$ désignent alors les coefficients de cette altraclion. L'invariabilité de $\Sigma \mathbf{M}$ dans les diverses transformations (variation de température, combinaison chimique, etc.) s'exprime, en langage ordinaire, par la loi de la conservation du poids de la matière. 